\title{
Characterisation of Au nanorod dynamics in optical tweezers via localised surface plasmon resonance spectroscopy
}

\begin{abstract}
Ana Andres-Arroyo, Scott Kemp, Wen Jun Toe, Fan Wang, Victoria Coleman, et al.
\end{abstract}

Ana Andres-Arroyo, Scott Kemp, Wen Jun Toe, Fan Wang, Victoria Coleman, Peter J. Reece, "Characterisation of Au nanorod dynamics in optical tweezers via localised surface plasmon resonance spectroscopy," Proc. SPIE 9164, Optical Trapping and Optical Micromanipulation XI, 91641I (16 September 2014); doi: 10.1117/12.2062465

SPIE Event: SPIE NanoScience + Engineering, 2014, San Diego, California, United States 


\title{
Characterisation of Au Nanorod Dynamics in an Optical Tweezers via Localised Surface Plasmon Resonance Spectroscopy.
}

\author{
Ana Andres-Arroyo ${ }^{a}$, Scott Kemp $^{a}$, Wen Jun Toe ${ }^{a}$, Fan Wang $^{a}$, Victoria Coleman ${ }^{b}$ and Peter \\ Reece $^{a}$ \\ ${ }^{a}$ School of Physics, The University of New South Wales, Sydney, NSW 2052, Australia \\ ${ }^{b}$ National Measurement Institute, Nanometrology Section, Lindfield, NSW 2070, Australia
}

\begin{abstract}
We present a study of the trapping properties of Au nanorods of different aspect ratios in an optical tweezers and comparison with other characterization techniques like transmission electron microscope (TEM) imaging and dynamic light scattering (DLS). This study provides information on the dynamics and orientation of Au nanorods inside an optical trap based on a time study of their localised surface plasmon resonance (LSPR) features. The results indicate that the orientation of the $\mathrm{Au}$ nanorods trapped in our optical tweezers varies with time and LSPR spectra can provide information on the angle of the nanorod with respect to the direction of propagation of the trapping laser.
\end{abstract}

Keywords: optical trapping, optical tweezers, nanorods, plasmonics

\section{INTRODUCTION}

Metal nanoparticles have many aplications in nanoscale sensing due to their unique size-dependent properties, such a localised surface plasmon resonances (LSPR). ${ }^{1}$ Using two-photon luminescence microscopy, gold nanorods have already been employed for in vitro and in vivo experiments ${ }^{2}$ and for labeling cancer cells. ${ }^{3}$ LSPR is responsible for the local electric field enhancement and strong scattering exhibited by metallic nanoparticles. The LSPR peak is strongly dependent on several factors, including particle size and shape and its local dielectric environment, making spectroscopy a sensitive probe of these features.

Optical tweezers can be used to isolate single micron and nano-sized objects and study their physical properties. Due to their strong polarizability, metallic nanoparticles are ideal for optical trapping. ${ }^{4}$ Gold spheres down to $18 \mathrm{~nm}$ in diameter ${ }^{5}$ have been successfully trapped in three dimensions. It has also been shown that it is possible to trap individual gold nanorods in an optical tweezers. ${ }^{6-8}$

In this paper we study the dynamics and orientation of gold nanorods inside an optical trap based on a time study of their LSPR features. We have observed that the orientation of the Au nanorods trapped in our optical tweezers varies with time and comparison between experimental spectra and Discrete Dipole Approximation (DDA) scattering calculations can potentially yield information on the angle of the nanorod with respect to the optical axis.

\section{MATERIALS AND METHODS}

We studied Au nanorods with lower aspect ratio (around 3.5 to 5), diameter of $12 \mathrm{~nm}$ and lengths of 42 to $60 \mathrm{~nm}$, and $\mathrm{Au}$ nanorods rods with higher aspect ratio (around 6 to 8), diameter of $15 \mathrm{~nm}$ and lengths of 90 to $120 \mathrm{~nm}$. Conventional techniques like transmission electron microscope (TEM) imaging and dynamic light scattering (DLS) were used to determine the particle dimensions. Optical tweezers can also be used to determine the dimensions of the nanorods using back focal plane interferometry in the same way Pearce et al. ${ }^{10}$ measure the diameter of trapped nanospheres.

A schematic diagram of the optical tweezers used for this study is presented in Fig 1. The optical tweezers comprise of a linearly polsrised 5W, CW Nd:YAG laser (Laser Quantum, 1064 Ventus, $\lambda=1064 \mathrm{~nm}$, TEM 00 ),

Further author information: (Send correspondence to Ana Andres-Arroyo.)

Ana Andres-Arroyo: E-mail: a.andresarroyo@unsw.edu.au

Peter Reece: E-mail: p.reece@unsw.edu.au

Optical Trapping and Optical Micromanipulation XI, edited by Kishan Dholakia,

Gabriel C. Spalding, Proc. of SPIE Vol. 9164, 91641I · () 2014 SPIE

CCC code: $0277-786 X / 14 / \$ 18 \cdot$ doi: $10.1117 / 12.2062465$

Proc. of SPIE Vol. $9164916411-1$ 


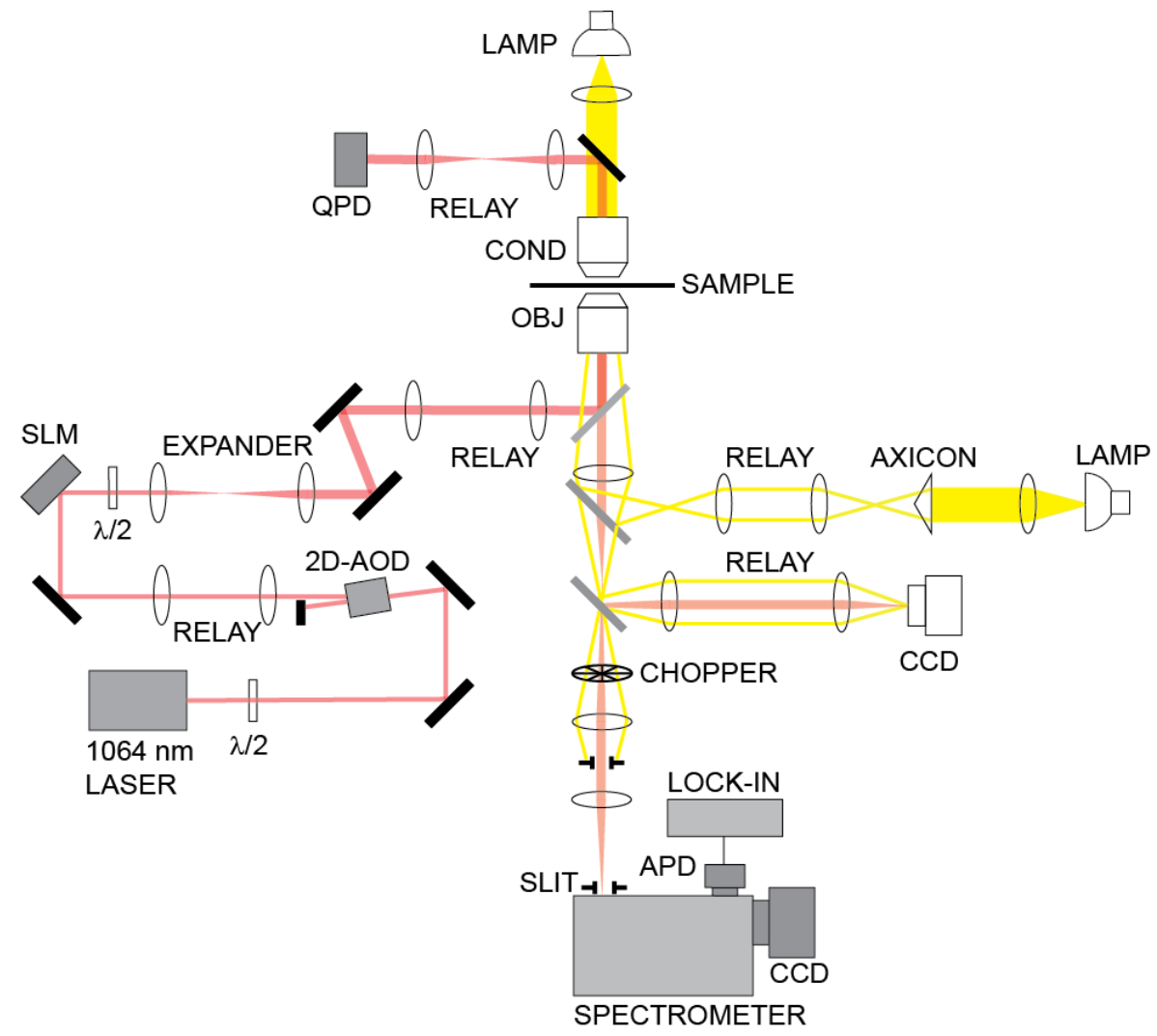

Figure 1. Optical tweezers setup that incorporates dark-field microscopy / spectroscopy. The illuminating light is formed into a ring by using an axicon. The image of this ring is projected onto the sample; its reflection is blocked from entering the CCD camera by using an aperture, allowing only the scattered light to pass.

which is focused using an oil-immersion microscope objective (Olympus, E-plan 100x, NA = 1.25), via relay optics, in order to create a diffraction limited spot in the front focal plane. A two-axis acousto-optic (AO) deflector (NEOS Technologies) provides automated control of the position of the laser within the objective's field-of-view with a fast response time. The AO deflectors are also used to control the power of the beam and a half-wave plate is used to control the polarization in the focal plane. The absolute power at the focus is measured using a calibrated photodetector taking into account the transmission losses of the optical system and microscope objective.

Dark field microscopy can be combined with optical tweezers to measure LSPR spectra on single isolated nanoparticles without compromising the strength of the optical trap. ${ }^{10}$ In our setup, we achieve dark field microscopy by using a backscattered light to image the sample. An axicon lens was used to form a ring on light which was imaged onto the back focal plane of the objective. The reflected righ was excluded from being collected by the CCD camera or spectrometer (Acton SP2300 \& PIXIS 256, Princeton Instruments) by placing a circular aperture before each of these elements. BFP interferometry can be implemented with the use of a 40x condenser lens with a NA of 0.6 (Olympus S Plan Fluor 40x 0.6NA) that collects the scattered trapping laser and projects it onto a quadrant photo-diode (QPD) (Electro Optical System InGaAs 030 QUAD) placed in the back focal plane of the condenser lens.

The nanorod samples were diluted with milli-Q water from stock solution to ensure that only one particle enters the tweezers during the course of the measurements. A $10 \mu \mathrm{L}$ voluyme of the nanoparticle suspension was pipetted into a sealed microfluidic chamber with a height of approximately $100 \mu \mathrm{m}$. Trapping measurements were performed on individual nanoparticles at a height of approximately $40 \mu \mathrm{m}$ from the cover slip. Trapping power at focus was $140 \mathrm{~mW}$. Dark-field spectra were collected through the spectrometer CCD (PIXIS 256) with an integration time of $1 \mathrm{sec}$ to observe the LSPR peak of the trapped nanorod. 

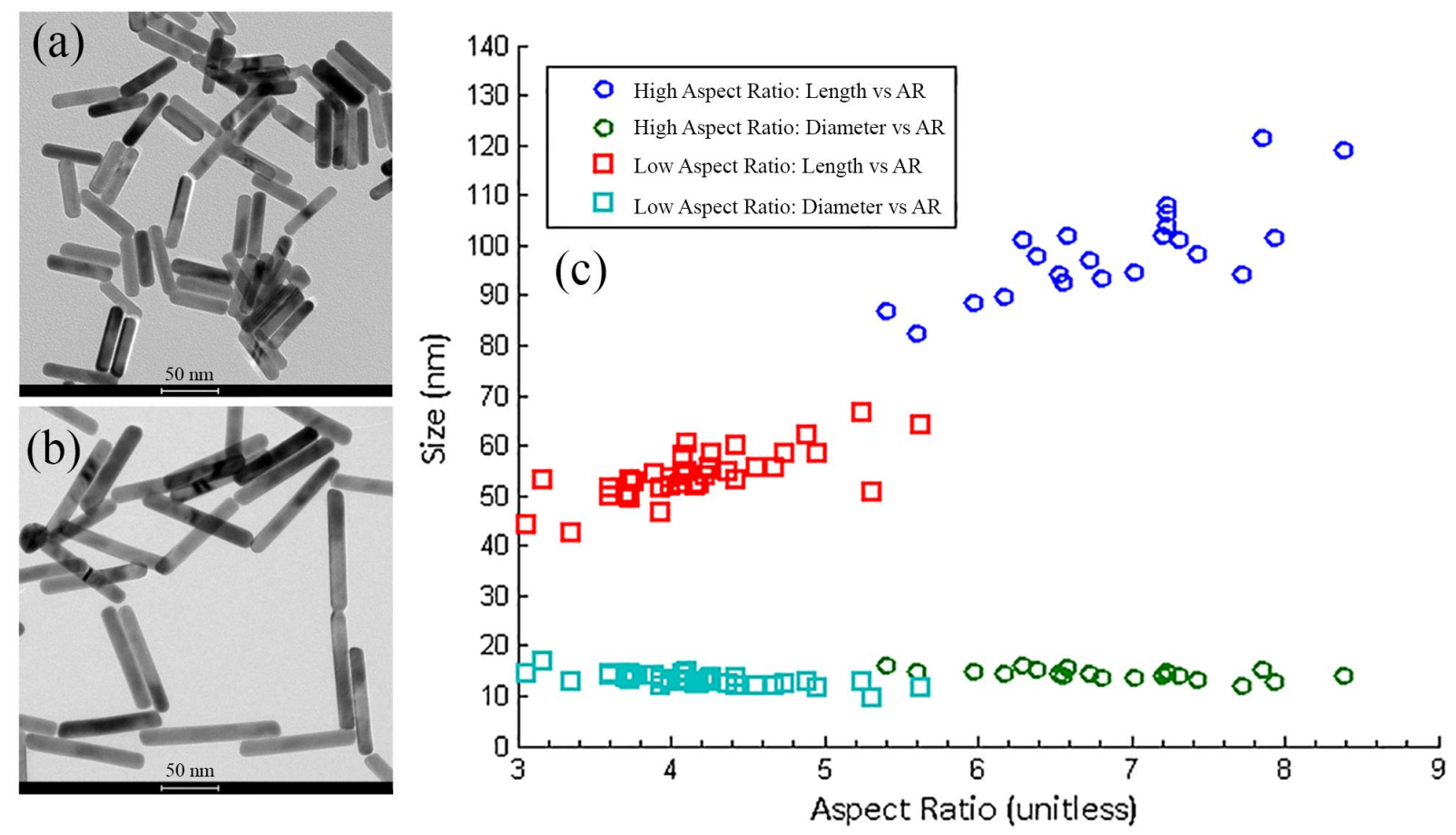

Figure 2. TEM images of the nanorods with lower aspect ratio (a), and nanorods with higher aspect ratio (b). Diameter and lenth versus aspect ratio of these Au nanorods (c). Lower aspect ratio nanorods (around 3.5 to 5) (squares) are measured to have diameters of $12 \mathrm{~nm}$ and lengths of 42 to $60 \mathrm{~nm}$, and higher aspect ratio nanorods (around 6 to 8) (circles) are measured to have diameters of $15 \mathrm{~nm}$ and lengths of 90 to $120 \mathrm{~nm}$.

\section{RESULTS AND DISCUSSION}

$\mathrm{Au}$ nanorods were imaged through a TEM and their dimensions were measured (figure 2). The lower aspect ratio nanorods (around 3.5 to 5) are measured to have diameters of $12 \mathrm{~nm}$ and lengths of 42 to $60 \mathrm{~nm}$, and the higher aspect ratio nanorods (around 6 to 8) are measured to have diameters of $15 \mathrm{~nm}$ and lengths of 90 to $120 \mathrm{~nm}$. DLS measurements for lower aspect ratio rods show lengths of $119 \mathrm{~nm}$ and aspect ratio of 4.73 . The aspect ratio closely agrees with the values obtained from the TEM imaging but the length differs by $50 \%$. This is probably due to the variation in longitudinal and rotational diffusion coefficients of the nanorods. Both DLS and BFP interferometry of trapped nanoparticles measure the hydrodynamic drag of the particles. DLS measures an average value for an ensemble whereas BFP interferometry on trapped nanoparticles allows single particle measurements. These techniques will result in somewhat different values for particle dimensions than other conventional techniques like TEM or atomic force microscopy (AFM) imaging.

The higher aspect ratio Au nanorods were trapped in our optical tweezers but the lower aspect ratio nanorods were not suitable for our optical tweezers and could not be trapped. Back scattered DF spectra were taken of the higher aspect ratio trapped Au nanorods. A higher scattering intensity was observed when the nanorod was freely diffusing in the water solution and a lower intensity when the nanorod was trapped in our optical tweezers. This leads us to believe that the initial trapping conditions for the nanorod show a preference for it being oriented along the axis of propagation of the trapping laser. However, even though the particle is trapped and it's translational motion is greatly reduced by the optical tweezers, it seems that optical forces cannot overcome the rotational Brownian motion of the nanorod. Hence, this nanorod can rotate inside the trap and, in some instances, sit on the trapping plane, as observed by Selhuber-Unkel et al. ${ }^{6}$

In figure 3 (b) we present LSPR back scattered DF spectra for a trapped nanorod. We can observe that the spectra varies over time, which indicates that the Au nanorod is oscillating with a varying angle $\theta$ with respect to the laser propagation axis. Since our DF illumination source is unpolarized white light we cannot measure any rotations of the nanorod within the trapping plane.

If the $\mathrm{Au}$ nanorod was aligned along the trapping laser propagation axis we would expect it's spectrum to have a single monomodal peak at a shorter wavelength. If the Au nanorod was aligned within the trapping 

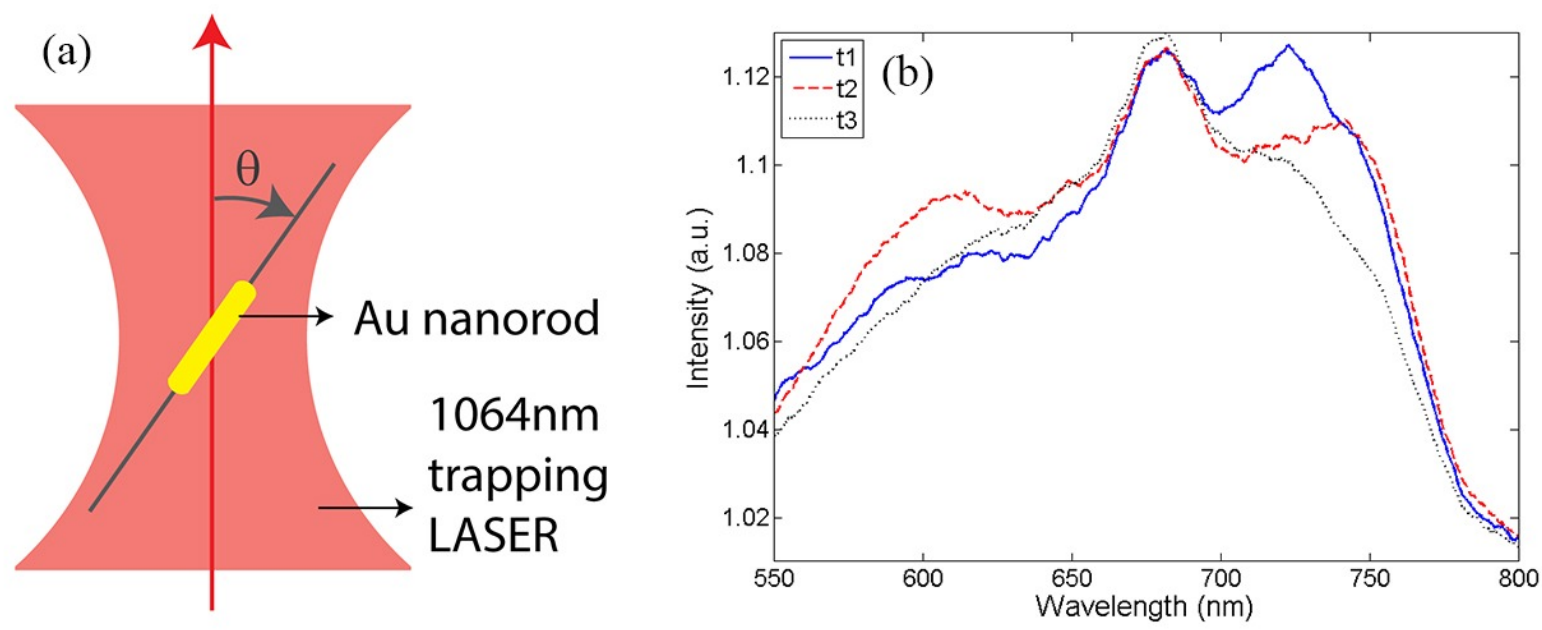

Figure 3. (a) Schematic diagram of the orientation of the nanorod in the optical trap. (b) LSPR spectra of nanorods in the optical for 3 different times. The different spectral shape depends on the angle of the nanorod.

plane we would expect it to be less confined and have a spectral peak in the IR region. However, our spectra observed are not monomodal and have two distinct peaks, leading us to believe that the nanorod is oriented with a varying angle $\theta$ with respect to the laser propagation axis. Discrete Dipole Approximation (DDA) scattering simulations can be done to better understand the effect of the nanorod orientation on it's scattering spectra, and provide a point of reference for comparison with experimental results.

\section{CONCLUSION}

Through DF spectroscopy we probe the trapping dynamics of higher aspect ratio Au nanorods (aspect ratios of 6 to 8). The differences in the scattering spectra observed for the same trapped nanorod at different times are on account of the optical forces of the trap not being able to overcome the rotational Brownian motion of the nanorod.

When the size of the trapped nanoparticle decreases the restoring torque and translational force scale different so perhaps it is possible that for small particles the restoring torque becomes negligible allowing the nanorod to freely orient within the trap while the restoring force is still strong enough to keep it trapped.

\section{REFERENCES}

[1] Anker, J. N., Hall, W. P., Lyandres, O., Shah, N. C., Zhao, J., and Van Duyne, R. P., "Biosensing with plasmonic nanosensors," Nature Materials 7(6), 442-453 (2008).

[2] Wang, H., Huff, T. B., Zweifel, D. A., He, W., Low, P. S., Wei, A., and Cheng, J. X., "In vitro and in vivo two-photon luminescence imaging of single gold nanorods," Proceedings of the National Academy of Sciences of the United States of America 102(44), 15752-15756 (2005).

[3] Durr, N. J., Larson, T., Smith, D. K., Korgel, B. A., Sokolov, K., and Ben-Yakar, A., "Two-photon luminescence imaging of cancer cells using molecularly targeted gold nanorods," Nano Letters 7(4), 941945 (2007).

[4] Svoboda, K. and Block, S. M., "Optical trapping of metallic rayleigh particles," Optics Letters 19(13), 930-932 (1994).

[5] Hansen, P. M., Bhatia, V. K., Harrit, N., and Oddershede, L., "Expanding the optical trapping range of gold nanoparticles," Nano Letters 5(10), 1937-1942 (2005).

[6] Selhuber-Unkel, C., Zins, I., Schubert, O., Snnichsen, C., and Oddershede, L. B., "Quantitative optical trapping of single gold nanorods," Nano Letters 8(9), 2998-3003 (2008).

[7] Pelton, M., Liu, M., Kim, H. Y., Smith, G., Guyot-Sionnest, P., and Scherer, N. F., "Optical trapping and alignment of single gold nanorods by using plasmon resonances," Optics Letters 31(13), 2075-2077 (2006).

[8] Toussaint Jr, K. C., Liu, M., Pelton, M., Pesic, J., Guffey, M. J., Guyot-Sionnest, P., and Scherer, N. F., "Plasmon resonance-based optical trapping of single and multiple au nanoparticles," Optics Express 15(19), 12017-12029 (2007). 
[9] Ashkin, A., Dziedzic, J. M., Bjorkholm, J. E., and Chu, S., "Observation of a single-beam gradient force optical trap for dielectric particles," Optics Letters 11(5), 288-290 (1986).

[10] Pearce, K., Wang, F., and Reece, P. J., "Dark-field optical tweezers for nanometrology of metallic nanoparticles," Optics Express 19(25), 25559-25569 (2011). 